\title{
Evidences of evanescent Bloch waves in phononic crystals
}

\author{
V. Romero-García, ${ }^{1, a)}$ J. V. Sánchez-Pérez, ${ }^{1}$ S. Castiñeira-Ibáñez, ${ }^{2}$ and \\ L. M. Garcia-Raffi ${ }^{3}$ \\ ${ }^{1}$ Centro de Tecnologías Físicas: Acústica, Materiales y Astrofísica, Universidad Politécnica de Valencia, \\ Valencia 46020, Spain \\ ${ }^{2}$ Dpto. Física Aplicada, Universidad Politécnica de Valencia, Valencia 46020, Spain \\ ${ }^{3}$ Instituto Universitario de Matemática Pura y Aplicada, Universidad Politécnica de Valencia, \\ Valencia 46020, Spain
}

(Received 7 January 2010; accepted 26 February 2010; published online 24 March 2010)

\begin{abstract}
We show both experimentally and theoretically the evanescent behavior of modes in the band gap of finite phononic crystal (PC). Based on experimental and numerical data we obtain the imaginary part of the wave vector in good agreement with the complex band structures obtained by the extended plane wave expansion. The calculated and measured acoustic field of a localized mode out of the point defect inside the PC presents also evanescent behavior. The correct understanding of evanescent modes is fundamental for designing narrow filters and waveguides based on PCs with defects. (C) 2010 American Institute of Physics. [doi:10.1063/1.3367739]
\end{abstract}

During the past few years, there has been a great deal of interest in studying propagation of waves inside periodic structures. These systems are composites made of inhomogeneous distribution of some material periodically embedded in other with different physical properties. Phononic crystals $(\mathrm{PC})^{1,2}$ are one of the examples of these systems. PCs are the extension of the so-called photonic crystals ${ }^{3}$ when elastic and acoustic waves propagate in periodic structures made of materials with different elastic properties. When one of these elastic materials is a fluid medium, then PC are called sonic crystals (SC). ${ }^{4,5}$

For these artificial materials, both theoretical and experimental results have shown several interesting physical properties. ${ }^{6}$ In the homogenization limit, ${ }^{7}$ it is possible to design acoustic metamaterials that can be used to build refractive devices. ${ }^{8}$ In the range of wavelengths similar to the periodicity of the PC $(\lambda \simeq a)$, multiple scattering process inside the PC leads to the phenomenon of so called band gaps (BGs), which are required for filtering sound, ${ }^{5}$ trapping sound in defects, ${ }^{9,10}$ and for acoustic wave guiding. ${ }^{11}$

Propagating waves inside a periodic media are a set of solutions of the wave equations satisfying the translational symmetry property. However, periodic media with point defects where the translational symmetry is broken, or finite periodic media, can support evanescent modes as well. Recently Laude et al. ${ }^{13}$ have analyzed the evanescent Bloch waves and the complex band structure of PC. Complex band structures show bands that are simply not revealed by the traditional $\omega(k)$ method. By means of the complex band structures, BG can be defined as ranges of frequencies where all Bloch waves must be evanescent.

The goal of the paper is to characterize the evanescent behavior of waves with frequencies in the BG inside of PC. Analytical, numerical, and experimental data show the evidences for the exponential-like decay of these modes. Engelen et al. ${ }^{12}$ shown that modes inside BG in photonic crystals decay multiexponentially. Supercell approximation in extended plane wave expansion (EPWE) ${ }^{13-16}$ has been used

${ }^{a)}$ Electronic mail: virogar1@mat.upv.es. in the present work to determine the imaginary part of the wave vector of evanescent modes. Specifically, we have deduced both analytically and experimentally the imaginary part of the wave vector observing that only the first harmonic contributes substantially to the decay of the acoustic field inside complete SC. In all cases we have obtained a very good agreement between theoretical and experimental results.

We have performed experiments in an echo-free chamber of dimensions $8 \times 6 \times 3 \mathrm{~m}^{3}$. The finite two-dimensional $\mathrm{SC}$ used in this paper forms a square array with lattice constant $a=22 \mathrm{~cm}$. The size of the SC is $5 a \times 5 a$ and the radius of the cylinders is $r=10 \mathrm{~cm}$. A prepolarized free-field 1/2" microphone Type 4189 B\&K has been used throughout the experiments. The diameter of the microphone is $1.32 \mathrm{~cm}$, which is approximately $0.06 a$. Our system 3DReAMS (three-dimensional Robotized e-Acoustic Measurement System) is capable of sweeping the microphone through a 3D grid of measuring points located at any trajectory inside the echo-free chamber. Motion of the robot is controlled by NI-PCI 7334.

Figure 1 shows the complex and real band structures for the SC with a point defect. The complex band structures and the value of the $k$ number for the modes inside the BG can be obtained by EPWE and it becomes in a purely real value for the localized mode. We can observe the localized mode at $920 \mathrm{~Hz}$ (continuous line). That value exactly coincides with the value obtained by PWE with supercell approximation. We have compared these results with experimental data measuring the insertion loss (IL) behind the SC with and without the point defect. In Fig. 1, we can observe that the experimental IL for the localized mode at frequency $920 \mathrm{~Hz}$ (continuous line) is lower than the case of the complete SC (dashed line), i.e., it can be concluded that there is a passing mode. This results because the localized mode is not killed completely by the SC around the point defect (see also Ref. 10). In fact, although the localized mode has an evanescent behavior, as we will see later, in this case there is not enough number of rows around the point defect to kill it.

For frequencies in the BG, the borders of the point defect act as perfect mirrors producing the localization in this 


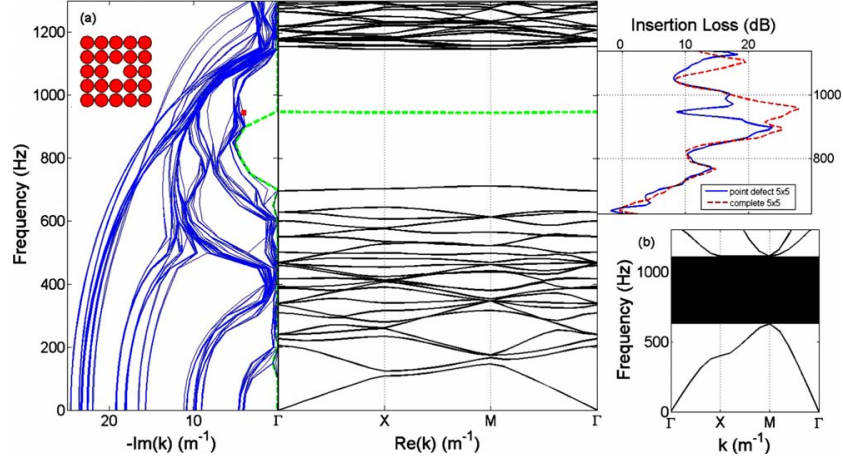

FIG. 1. (Color online) Band structures of a complete $\mathrm{SC}$ and of a SC with a defect point vs experimental data for a complete SC and a SC with a point defect. (a) Left panel: Complex Band Structure calculated by EPWE with the supercell approximation. Central panel: Real Band Structure. Dashed line represents the localized mode. Right panel: Experimental Insertion Loss of the complete SC (dashed line) in the Band Gap and Experimental Insertion Loss of the SC with a point defect (continuous line). The inset shows the supercell used in the calculations. Red square marks the value of the imaginary part of the wave vector $\operatorname{Im}(k)=-5.6$. (b) Band structures for a complete SC. SC made of PVC cylinders with $r=0.1 \mathrm{~m}, \rho_{\mathrm{PVC}}$ $=1400 \mathrm{~kg} / \mathrm{m}^{3}$ and $c_{\mathrm{PVC}}=2380 \mathrm{~m} / \mathrm{s}$, embedded in air, $\rho_{\text {air }}=1.23 \mathrm{~kg} / \mathrm{m}^{3}$ and $c_{\text {air }}=340 \mathrm{~m} / \mathrm{s}$.

cavity. ${ }^{6}$ The localized mode sees a complete SC from inside the point defect in every directions. Thus, the localized mode should present, out of the cavity, a decay analogous to a wave with the same frequency impinging over the SC from outside. Both cases should be represented by the same $\operatorname{Im}(k)$, i.e., by the same evanescent behavior. There are several values of the imaginary part of the wave vector at the localized frequency in the complex band structures shown in Fig. 1 which shows the multiexponential behavior. In this paper, we show for the localized frequency, $920 \mathrm{~Hz}$, that the first harmonic obtained from the Fig. 1 (solid square), $\operatorname{Im}(k)=-5.6 \mathrm{~m}^{-1}$, can represent the decay of the mode inside the SC. The contribution to the next harmonics to the multiexponential decay can be neglected.

In order to study this behavior of the localized mode we have analyzed numerically the acoustic field inside the SC.
In Figs. 2(a) and 2(b) we can observe the maps obtained by finite element method (FEM) for the complete SC and for the SC with a point defect, respectively. In Fig. 2(c), we represent both numerical and experimental absolute values of the pressure for complete SC and for SC with a point defect corresponding to the cross sections marked with a red line in Figs. 2(a) and 2(b). Experimental results are also plotted in Fig. 2(c).

For a complete SC, we can observe in Fig. 2 both numerically (dashed continuous) and experimentally (open circles) exponential-like decay of the mode with the distance all along the SC without point defect. From these experimental data, we have chosen the points with maximum values in order to fit an exponential $a e^{b x}$. The values of the parameters in the fit are $a=0.02938 \pm 0.0103$ and $b=\operatorname{Im}(k)$ $=-5.60 \pm 1.45 \mathrm{~m}^{-1}$.

In the blue line in Fig. 2(c) we can observe the effect of the point defect in the acoustic field inside the SC. In the region of the point defect there is an increasing value of the acoustic pressure because of the localized mode. We can also observe that the absolute value of the pressure for the localized mode is bigger than in the case of the complete SC at the end of the SC, proving the passing mode shown in Fig. 1. To enhance the localization of the sound inside the SC we would need a SC with a bigger number of rows around the point defect as it was shown elsewhere. ${ }^{10}$

The border of the cavity is located approximately at $x=0.6 \mathrm{~m}$ as we can observe in Fig. 2(b). From this point to the end of the SC we can observe that the acoustic field is drastically reduced but with these evidences we cannot confirm that the behavior of the localized mode out of the cavity is evanescent. To do that, we have analyzed the sound inside a bigger SC with a point defect [see inset of Fig. 3(a)]. Figure 3(a) presents both numerical (blue line) and experimental (blue open circles) values of the acoustic field from the end of the cavity to the end of a SC showing the evanescent behavior of the localized mode out of the cavity. Analogously as the case of complete SC, here we have also chosen the points with maximum values [see black open circles in (a)

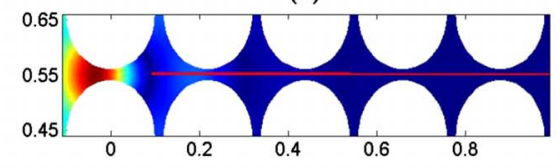

(c)

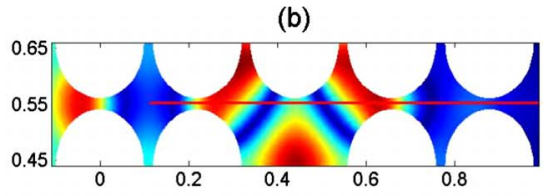

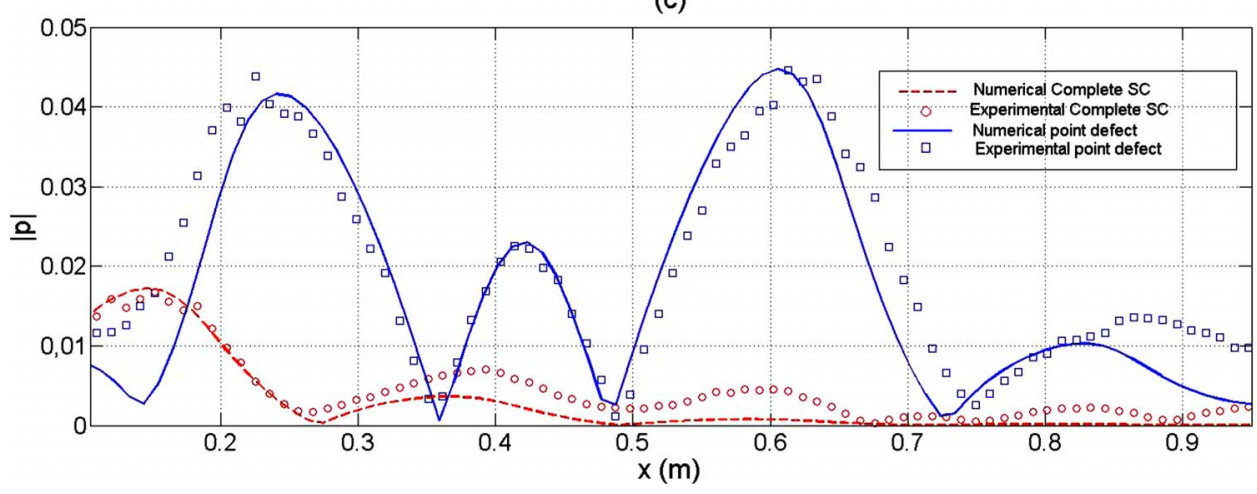

FIG. 2. (Color online) Absolute values of the acoustic field inside the SC with and without point defect. Numerical maps calculated by FEM inside the complete SC (a) and inside the SC with a point defect (b). (c) Numerical and Experimental data for the interior path marked in [(a) and (b)] with a continuous line. Dashed line (open circles) represents the numerical (experimental) results for complete SC. Continuous line (open squares) represents the numerical (experimental) results for SC with a point defect. 

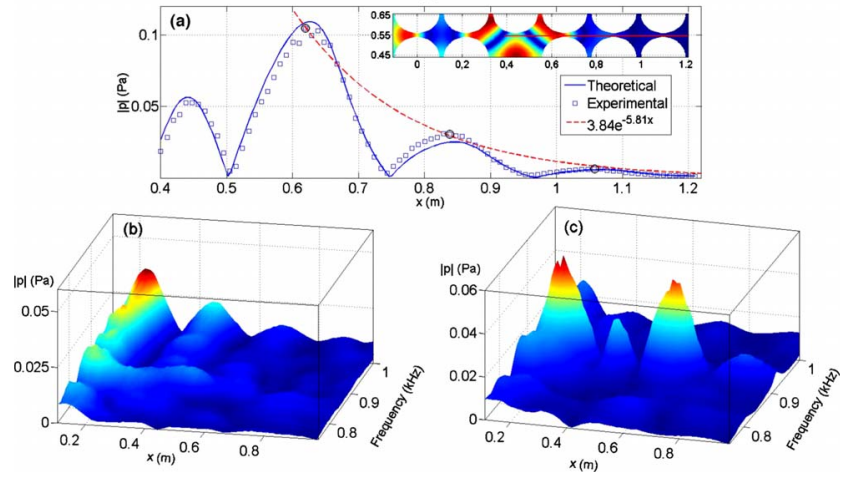

FIG. 3. (Color online) (a) Absolute values of pressure inside a $6 \times 5 \mathrm{SC}$ with a point defect: Numerical results (continuous line), experimental results (open squares). Dashed line represents the fitted exponential-like decay of the localized mode using the open circles. (b) and (c) represent the 3D spectra for the complete SC and for the SC with a point defect, respectively.

Fig. 3(a)] in order to fit an exponential decay $a e^{b x}$. The values of the parameters in the fit are $a=3.84 \pm 9.92$ and $b=\operatorname{Im}(k)=-5.81 \pm 4.06 \mathrm{~m}^{-1}$ and the curve is also plotted in Fig. 3(a) with red dashed line. From the experimental point of view we are constrained by the size of the SC, and as a consequence we have only been able to use a few points for the exponential fit. This results in a big error in the parameters of the fit. Even so, the value obtained for the $\operatorname{Im}(k)$ is very closed to the one obtained both analytically (EPWE) and experimentally for the complete SC. The difference is less than $4 \%$ in both cases.

We have analyzed one frequency $(920 \mathrm{~Hz})$ inside the BG but this exponential-like decay should be observed for every frequency inside $\mathrm{BG}$ independently if there is or not a point defect in the SC. Figures 3(b) and 3(c) represent the experimental 3D spectra for both complete SC and SC with a point defect, respectively, in the range of frequencies 750-1000 $\mathrm{Hz}$, in the BG. In Fig. 3(b), we observe the experimental evidences of the evanescent behavior for all the modes inside the BG for a complete SC. For the case of the SC with a point defect there is a change in the propagation properties. We observe clearly in Fig. 3(c) the evanescent behavior of the localized frequencies out of the region of the point defect and how the localization is produced, showing the effect of the cavity. ${ }^{10}$ We can also observe in Fig. 3(c) the evanescent behavior for all modes out of the frequencies of the localization range.

The propagation of waves inside periodic structures consists on both propagating and evanescent modes. Using EPWE we have observed the evanescent nature of the modes inside the BG with negative complex Bloch vectors.
From the experimental point of view we have observed that the exponential-like decay is dominated by the first harmonic of the Fourier expansion of the Bloch wave, obtaining this exponential-like decay for the modes in the BG $[\operatorname{Im}(k)$ $=-5.6 \pm 1.45 \mathrm{~m}^{-1}$ for $\left.920 \mathrm{~Hz}\right]$ and for localized modes in a $\mathrm{SC}$ with a point defect $\left[\operatorname{Im}(k)=-5.81 \pm 4.06 \mathrm{~m}^{-1}\right]$. From analytical and experimental data we can conclude that localized modes present evanescent behavior out of the cavity with the same exponential-like decay as a wave with the same frequency impinging over a complete SC. Due to the breaking periodicity, the physical situations are very different between complete SC and SC with point defects, even so we can conclude that the space observed by the localized wave from inside of the cavity is topologically equivalent to the observed by the same wave from outside of a complete SC. This work is fundamental for the correct understanding of the design of narrow filters and waveguides based on PCs with point defects.

This work was supported by MEC (Spanish Government) and FEDER funds, under Grant Nos. MAT2009-09438 and MTM2009-14483-C02-02. The authors would like to thank J. M. Herrero, S. García-Nieto, and X. Blasco for their work in the control and acquisition system of 3DReAMS.

${ }^{1}$ M. Sigalas and E. Economou, Solid State Commun. 86, 141 (1993).

${ }^{2}$ M. S. Kushwaha, P. Halevi, G. Martínez, L. Dobrzynski, and B. DjafariRouhani, Phys. Rev. B 49, 2313 (1994).

${ }^{3}$ E. Yablonovitch, Phys. Rev. Lett. 58, 2059 (1987).

${ }^{4}$ R. Martínez-Sala, J. Sancho, J. V. Sánchez, V. Gómez, J. Llinares, and F. Meseguer, Nature (London) 378, 241 (1995).

${ }^{5}$ J. V. Sánchez-Pérez, D. Caballero, R. Martínez-Sala, C. Rubio, J. SánchezDehesa, F. Meseguer, J. Llinares, and F. Gálvez, Phys. Rev. Lett. 80, 5325 (1998).

${ }^{6}$ J. D. Joannopoulus, S. G. Johnson, J. N. Winn, and R. D. Meade, Photonic Crystals. Molding the Flow of Light (Princeton University Press, Princeton, 2008).

${ }^{7}$ D. Torrent, A. Hakansson, F. Cervera, and J. Sánchez-Dehesa, Phys. Rev. Lett. 96, 204302 (2006).

${ }^{8}$ D. Torrent and J. Sánchez-Dehesa, New J. Phys. 9, 323 (2007).

${ }^{9}$ F. Wu, Z. Hou, Z. Liu, and Y. Liu, Phys. Lett. A 292, 198 (2001).

${ }^{10}$ L. Wu, L. Chen, and C. Liu, Physica B 404, 1766 (2009).

${ }^{11}$ J. O. Vasseur, P. A. Deymier, B. Djafari-Rouhani, Y. Pennec, and A. C. Hladky-Hennion, Phys. Rev. B 77, 085415 (2008).

${ }^{12}$ R. J. P. Engelen, D. Mori, T. Baba, and L. Kuipers, Phys. Rev. Lett. 102, 023902 (2009).

${ }^{13}$ V. Laude, Y. Achaoui, S. Benchabane, and A. Khelif, Phys. Rev. B 80, 092301 (2009).

${ }^{14}$ V. Romero-García, J. V. Sánchez-Pérez, and L. M. Garcia-Raffi, arXiv:1001.3758v1 (unpublished).

${ }^{15}$ Y.-C. Hsue, A. J. Freeman, and B.-Y. Gu, Phys. Rev. B 72, 195118 (2005).

${ }^{16}$ A. Khelif, A. Choujaa, B. Djafari-Rouhani, M. Wilm, S. Ballandras, and V. Laude, Phys. Rev. B 68, 214301 (2003). 